\title{
Influence du mode de séchage et de la finesse de broyage des échantillons de fourrages sur l'estimation de la dégradabilité de l'azote dans le rumen
}

\author{
JL Peyraud \\ INRA, Station de Recherches sur la Vache Laitière, St-Gilles, 35590 L'Hermitage, France
}

Summary - The effects of the drying method (freeze-drying, forced-air oven at $60^{\circ} \mathrm{C}$ for $24 \mathrm{~h}$ and conventional oven at $80^{\circ} \mathrm{C}$ for $48 \mathrm{~h}$ ) and grinder screen size (0.8 vs $1.5 \mathrm{~mm}$ ) upon in sacco degradability were compared with 7 forages. Nitrogen degradability was reduced by oven drying even at the lower temperature, but screen size was less important.

Introduction - La congélation (MacRae et al, 1975) et la technique de préparation des échantillons de fourrage (Abdalla et al, 1988) entrainent des modifications physico-chimiques des constituants azotés et peuvent donc influencer la dégradabilité estimée de l'azote dans le rumen. Le but de ce travail a été d'étudier l'effet du mode de séchage et de la finesse de broyage des fourrages sur la mesure de la dégradabilité in sacco de l'azote dans le rumen.

Matériel et Méthodes - L'étude a porté sur 7 fourrages conservés depuis leur prélèvement par congélation à $-15^{\circ} \mathrm{C}$ et choisis pour avoir une gamme de dégradabilité théorique (DT) comprise entre 0,70 et $0,90: 2$ ensilages de trèfle violet (TV) (43 j de repousses, avec et sans acide formique), 3 ensilages de graminées récoltés juste avant l'épiaison (avec et sans conservateur), un ensilage de maïs et un brome récolté en vert au stade épiaison. Pour chacun d'eux nous avons comparé 6 traitements : séchage par lyophilisation $(L)$, à $60^{\circ} \mathrm{C}$ pendant 24 $h$ en étuve fortement ventilée (E60) ou à $80^{\circ} \mathrm{C}$ pendant $48 \mathrm{~h}$ en étuve faiblement ventilée (E80) puis broyage sur une grille de 0,8 ou de 1,5 $\mathrm{mm}$. Le taux de disparition in sacco de l'azote a été mesuré selon le protocole défini par Michalet-Doreau et al (1987) à partir de 6 points de cinétique $(2,4,8,16,24$ et $48 \mathrm{~h})$ avec 3 répéti- tions ( 3 vaches) sauf pour le séchage à $80^{\circ} \mathrm{C}$ où seuls les points 2,8 et $48 \mathrm{~h}$ ont été retenus. On a testé un aliment par jour en introduisant dans chacune des 3 vaches les 30 sachets correspondant au 6 traitements. L'azote a été dosé sur les aliments séchés et broyés en 0,8 $\mathrm{mm}$ ainsi que sur les résidus de chacun des points de cinétique. La DT de l'azote a été calculée par la méthode pas à pas (MichaletDoreau et al, 1987) et les données ont été analysées selon le modèle : $Y=$ moy + 'alim' + 'séchage' + 'broyage' + alim $x$ sec + alim $x$ broy + écart type résiduel (ETR).

Résultats et Discussion - La technique de séchage n'a pas eu d'influence sur la teneur en azote total des aliments qui a été, en moyenne pour les 7 aliments, de 2,59 (L), 2,55 (E60) et 2,52\% de la MS (E80). Seule la teneur en azote de l'ensilage de TV sans conservateur a été légèrement plus faible après un séchage à $80^{\circ} \mathrm{C}(3,31$ vs 3,49 pour $L)$, sans doute par suite des pertes en produits volatils. La DT de l'azote a été plus faible pour les 2 modes de séchage en étuve (tableau I). L'écart a été particulièrement important (13 points en moyenne) pour les échantillons séchés à $80^{\circ} \mathrm{C}$. Cette méthode ne peut donc pas être retenue pour la me- 
Tableau I. Influence du mode de séchage et de la finesse de broyage sur la mesure de la dégradabilité in sacco de l'azote des fourrages.

\begin{tabular}{|c|c|c|c|c|c|c|}
\hline & \multirow{2}{*}{$\begin{array}{l}N^{1} \\
(\% M S)\end{array}$} & \multicolumn{3}{|c|}{ Séchage } & \multicolumn{2}{|c|}{ Broyage } \\
\hline & & $L$ & $E 60$ & $E 80$ & $0,8 \mathrm{~mm}$ & $1,5 \mathrm{~mm}$ \\
\hline Ensilage TV & 3,49 & 81,7 & 76,5 & 68,5 & 75,9 & 75,6 \\
\hline Ens TV + ac formique & 3,42 & 81,0 & 75,1 & 65,5 & 74,3 & 73,7 \\
\hline Ens ray grass hybride & 2,16 & 81,3 & 77,3 & 65,3 & 75,6 & 73,9 \\
\hline+ conservateur & 3,01 & 89,1 & 85,8 & 82,1 & 86,2 & 85,4 \\
\hline Ens dactyle + conservateur & 3,13 & 85,0 & 83,0 & 74,3 & 82,0 & 79,9 \\
\hline Ens maîs & 1,29 & 80,4 & 76,0 & 69,1 & 75,7 & 74,9 \\
\hline Brome vert (épiaison) & 1,59 & 73,9 & 59,8 & 55,9 & 63,5 & 63,7 \\
\hline Moyenne ${ }^{2}$ & & 81,8 a & $76,2 \mathrm{~b}$ & $68,7 \mathrm{c}$ & 76,2 a & $75,3 \mathrm{~b}$ \\
\hline
\end{tabular}

${ }^{1}$ Sur l'échantillon lyophilisé. ${ }^{2}$ a, b, c : traitements différents $(P<0,01) ; E T R$ de l'analyse $=1,0$.

sure de la dégradabilité. L'écart de DT entre $L$ et $E 60$ a été plus modéré et a varié de 2 (ensilage de maïs) à 6 points (ensilage de TV) pour les fourrages conservés mais a atteint 14 points pour le seul fourrage vert testé (interaction alim $x$ sec significative $(P<0,01))$. L'écart entre ces 2 méthodes est surtout important en début de cinétique (10 points de différence à 2 et $4 \mathrm{~h}$ ) et se réduit ensuite rapidement (4 points à $8 \mathrm{~h}$, écarts négligeables ensuite). Ces résultats peuvent s'expliquer par la diminution de la solubilité de l'azote généralement observée après un traitement, même modéré, à la chaleur (Abdalla et al, 1988) mais aussi, notamment pour le fourrage vert, par une baisse de la solubilité consécutive à la décongélation subie par les échantillons dans l'étuve, ainsi que le suggèrent les données de MacRae et al (1975). La finesse de broyage a eu un effet significatif $(P<0,01)$, mais faible en regard du mode de séchage $(75,3$ vs 76,2 respectivement pour les grilles de 1,5 et de $0,8 \mathrm{~mm}$ ) et son effet n'a pas été diffé- rent, que le fourrage soit lyophilisé ou séché en étuve.

La précision des mesures, estimée par ETR de l'analyse de variance prenant en compte l'effet aliment et l'effet vache, a été peu différente entre les méthodes (ETR 1,1 et 0,7 avec $L$ respectivement pour 0,8 $\mathrm{mm}$ et $1,5 \mathrm{~mm} ; 1,5$ et 1,1 avec E60 pour 0,8 et $1,5 \mathrm{~mm} ; 1,9$ et 1,5 avec E80 pour 0,8 et $1,5 \mathrm{~mm}$ ).

En conclusion, le séchage à $80^{\circ} \mathrm{C}$ ne peut pas être retenu; la méthode E60 conduit à une DT inférieure à la lyophilisation. L'écart est variable selon les aliments mais il n'a pas été possible de faire la part de l'effet propre de la congélation et du mode de séchage des échantillons. Le broyage à $0,8 \mathrm{~mm}$ devrait pouvoir être adopté pour les fourrages.

Abdalla HO, Fox DG, van Soest PJ (1988) J Anim Sci 66, 2646-2649

MacRae JC, Campbell DR, Eadie J (1975) J Agric Sci 84, 125-131

Michalet-Doreau $B$, Vérité $R$, Chapoutot $P$ (1987) Bull Tech CRZV Theix, INRA 69, 5-7 